\title{
Comparing the lower limb strength after total and unicondylar knee arthroplasty
}

\begin{abstract}
Aims: The aim of this study was to examine the lower limb strength and knee function after total knee arthroplasty (TKA) and unicondylar knee arthroplasty (UKA).

Patients: In this prospective cohort study a total of 240 patients were evaluated who underwent a knee Arthroplasty. Of these patients 180 underwent a TKA and 60 a UKA. The study population consisted of 146 women and 94 men with a mean age of 64 years.

Methods: Measurements were done preoperative and subsequently;2 weeks, 8 weeks, 24 weeks and 1 year after the surgical procedure. Clinical measurements consisted ofa VAS score, Oxford Knee Score, range of motion (ROM) and the isometric strength of the lower limb.

Results: One year after the procedure a significant reduction of the VAS score was observed after both procedures (TKA \& UKA). Regarding the ROM of the knee, there was no significant change detectable. Regarding the isometric strength of the lower limb, there was a significant difference in favour of the UKA one year after the procedure. Regarding the Oxford Knee Score both the UKA and TKA showed a significant improvement.
\end{abstract}

Conclusion: Both UKA and TKA are successful procedures, but it seems that patients with an UKA have a significant better lower limb strength, one year after the surgical procedure, compared those who received a TKA.
Volume I Issue I - 2017

\author{
Muradin I, Breugem SJM, Breemans E, Bruijn \\ DJ, Driessen MJM \\ Orthopedium Clinics, Netherlands
}

Correspondence: Muradin I, Orthopedium Clinics, Netherlands, Tel 003I-88 0088 444,

Email sbreugem@orthopedium.nl

Received: May 14, 2017 | Published: May 22, 2017

\section{Introduction}

The prevalence of gonarthrosis is increasing promptly, as of today osteoarthritis is currently one of the leading causes of disability in not only elderly, but also young and active patients. ${ }^{1}$ Due to pain, instability and stiffness of the joints patients with gonarthrosis are progressively restricted in their daily functioning, making them less active. ${ }^{1,2}$ Although the TKA is a common successful surgical procedure, ${ }^{3}$ the postoperative outcome concerning the physical function, social function and pain reduction are not as well restored as seen in patients after total hip Arthroplasty. ${ }^{4}$ A recent systematic review, observed the isometric quadriceps strength after TKA; even 3 years after the procedure, patients still showed reduced strengths of the lower limb.

Besides the TKA, there is a rising popularity of the UKA for treating anteromedial or lateral gonarthrosis. ${ }^{5}$ The effects of UKA on the strength of the lower limbs has been examined and revealed an increase (70.4 Nm Newton meter) in the maximal voluntary contraction of the quadriceps muscle of the ipsilateral leg 18 months postoperative. ${ }^{6}$ Not only the contraction has been examined; Baker et al. discovered that the leg extensor power of patients increased (with $0.50 \mathrm{~W} / \mathrm{kg}$ ) one year after the UKA m. ${ }^{7}$

In light of the existing lack of evidence it was the aim of this study to compare the change of the lower limb strength after a UKA and TKA, one year after the procedure. This could be a possible explanation why patients after a UKA are more likely to return to (strength demanding) sports than patients that undergo TKA.'

\section{Materials and methods}

Inclusion: This prospective cohort study included 240 patients who were scheduled for aunicondylarortotal knee arthroplasty, with the indication of primary gonarthrosis according to the criteria of the American Rheumatism Association. ${ }^{8}$

Surgery and postoperative management: All procedures were done in ourclinic by 3 orthopedic surgeons. The surgical procedure was performed under tourniquet using the minimal invasive approach. The wound was closed using staples with appliance of a single intraarticular suction drain. For the total knee Arthroplasty the Genesis II (Smith and Nephew, Memphis, USA) was used in 30 patients and the Advanced Coated System (Implant cast GmbH, Buxtehude, Germany) in 150 patients. Both types were implanted using a medial parapatellar approach and cemented insertion of the femur and tibia component. For the Unicondylar knee Arthroplasty we used the Oxford Partial Knee Arthroplasty (Phase III, Biomet, Warsaw, IN, USA) was used, with non-cemented implementation. After the procedure people were encouraged to mobilize directly. Compression bandage was applied during the first 24hours and the suction drain was removed after 24 hours. Postoperative pain management was done using the LIA technique. ${ }^{8}$ Two weeks after the procedure the staples were removed and the patients started with routine physiotherapy.

Clinical evaluation: All patients were evaluated preoperative, 2 weeks, 8 weeks, 6 months and after one year. Pain was evaluated using the VAS score ranging from 0 to 100 , where a higher score reflects more pain. The patients perceived outcome was measured using the 
Oxford Knee Score, which consists of 12 questions covering function and pain associated with the knee. ${ }^{9}$ The range of motion (ROM) was measured by the physiotherapist. The ROM was estimated with the use of a goniometer, subsequently preoperative, 2 weeks, 8 weeks, 6 months and1 year after the procedure.

To evaluate the isometric strength of the lower limb, a horizontal pivotingclosed chain leg press system (Functional Squat System, Monitored Rehab Systems, Haarlem, and The Netherlands) was used toreplicate a standing squat. ${ }^{10}$ The patient was positioned in a supine position, with the hip and knee flexed at ninety degrees. Out of this position the patient was encouraged to slide upwards with maximal force on the fixated seat. The strength was measured and registered using an electronic pressure board located underneath the feet. These measurements were done preoperative and subsequently 8 weeks, 6 months and 1 year after the procedure.

Statistics: Data was analyzed using the statistical package SPSS version 23(SPSS, Chicago Ill, USA). Data are presented as mean with range and standard deviation (SD). Paired samples t-tests and one way ANOVA were used to compare continuous variables between the measurements. Significance levels were set at 0,05.

\section{Results}

Patients: The study population consisted of 240 patients undergoing a primary knee Arthroplasty. A total of 60unicondylar and 180 total knees Arthroplasty were included. Within the TKA group; the mean age was: 65 years (range: 41-86) within a group of 68 males and 112 females, contained 93 left knees and 87 right knees, in 169 cases the cruciate retaining TKP was used and in 11 cases the posterior stabilized TKP. Within the UKA group; there were 26 male and 34 female with a mean age of: 61 years (range: $47-79$ ), furthermore the group contained 31 left knees and 29 right knees.

VAS Score: Preoperative there was no significant difference in VAS score between the two groups $(\mathrm{p}=0.31)$. One year after the procedure there was neither a significant difference noticeable $(p=0.62)$ between the groups (Figure 1, Table 1). Paired sampled t-test showed for both procedures a significant reduction of the VAS score (UKA; $\mathrm{p}=0.000$, TKA: $\mathrm{p}=0.000$ ).

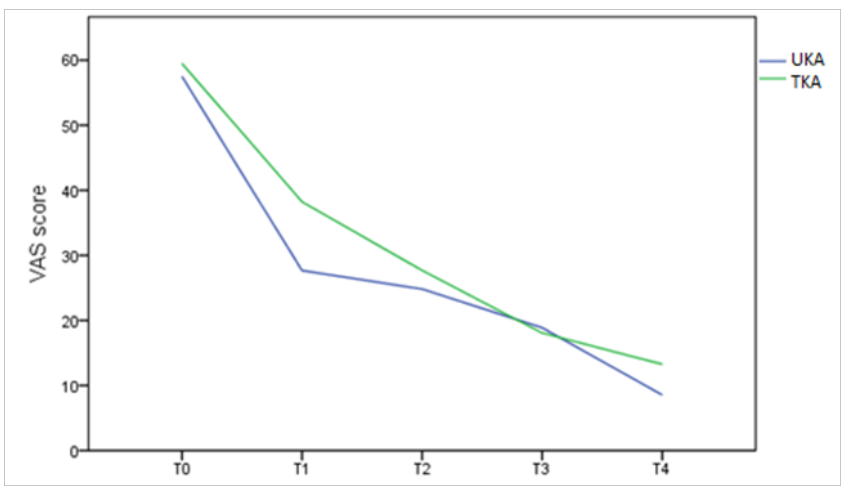

Figure I VAS score, during multiple measuring moments $(\mathrm{T} 0=$ preoperative, $\mathrm{TI}=2$ weeks, $\mathrm{T} 2=8$ weeks, $\mathrm{T} 3=6$ months, $\mathrm{T} 4=\mathrm{I}$ year ).

\section{Knee flexion}

Preoperative there was a significant difference between the groups $(p=0.000)$ concerning the degrees of flexion of the knee. One year after the procedure there was a significant difference noticeable $(\mathrm{p}=0.000)$ between the groups (Figure 2, Table 2). Paired sampled t-test showed for both procedures no significant change of the knee flexion in comparison to the preoperative flexion. (UKA; $\mathrm{p}=0.076$, TKA: $\mathrm{p}=0.287$ ).

Table I Descriptive statistics of the VAS score preoperative (T0) and one year after the procedure (T4)

\begin{tabular}{llllll}
\hline & $\mathbf{T}^{0}$ & $\mathbf{T}^{4}$ & $\mathbf{T}^{0}$ & $\mathbf{T}^{4}$ \\
UKA & & & TKA & & \\
\hline $\mathrm{N}$ & 60 & 60 & 180 & 180 \\
Minimum & 3 & 0 & 0 & 0 \\
Maximum & 100 & 70 & 100 & 80 \\
Mean & 57 & 11 & 60 & 12 \\
SD & 23 & 17 & 24 & 19 \\
\hline
\end{tabular}

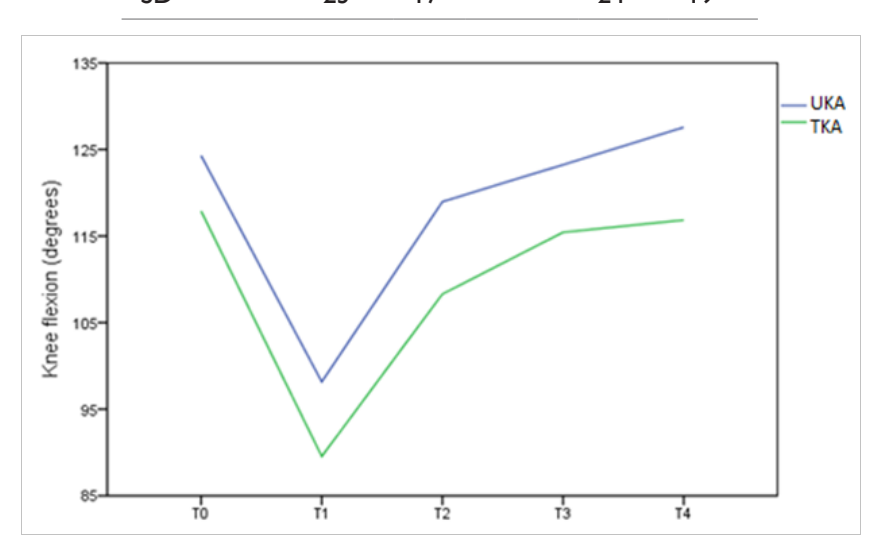

Figure 2 Knee flexion expressed in degrees, during multiple measuring moments $(\mathrm{T} 0=$ preoperative, $\mathrm{TI}=2$ weeks, $\mathrm{T} 2=8$ weeks, $\mathrm{T} 3=6$ months, $\mathrm{T} 4=$ Iyear).

Table 2 Descriptive statistics of the knee flexion preoperative (T0) and one year after the procedure (T4)

\begin{tabular}{llllll}
\hline & $\mathbf{T}^{0}$ & $\mathbf{T}^{4}$ & & $\mathbf{T}^{0}$ & $\mathbf{T}^{4}$ \\
UKA & \multicolumn{3}{c}{ TKA } \\
\hline $\mathrm{N}$ & 60 & 60 & & 180 & 180 \\
Minimum & $100^{\circ}$ & $110^{\circ}$ & $70^{\circ}$ & $95^{\circ}$ \\
Maximum & $145^{\circ}$ & $140^{\circ}$ & $160^{\circ}$ & $140^{\circ}$ \\
Mean & $124^{\circ}$ & $126^{\circ}$ & $117^{\circ}$ & $116^{\circ}$ \\
SD & $11^{\circ}$ & $7^{\circ}$ & & $13^{\circ}$ & $9^{\circ}$ \\
\hline
\end{tabular}

\section{Knee extension}

Preoperative there was no significant difference between the groups ( $\mathrm{p}=0.098$ ) regarding the extension of the knee. One year after the procedure there was similarly no significant difference noticeable $(\mathrm{p}=0.224)$ between the groups (Figure 3, Table 3). Paired sampled t-test showed for the UKA no significant change of the knee extension (UKA; $\mathrm{p}=0.037)$ and for the TKA a significant improvement $(\mathrm{p}=$ $0.000)$.

\section{Isometric squad test}

Preoperative there was no significant difference between the groups during the isometric squad test $(p=0.34)$. One year 
postoperative there was a significant difference noticeable between the groups ( $\mathrm{p}=0.000$ ), in favor of the UKA (Figure 4, Table 4). Paired sampled t-test showed for both procedures significant improvement, comparing the preoperative and postoperative strength (UKA; p:0.000, TKA:p:0.001).

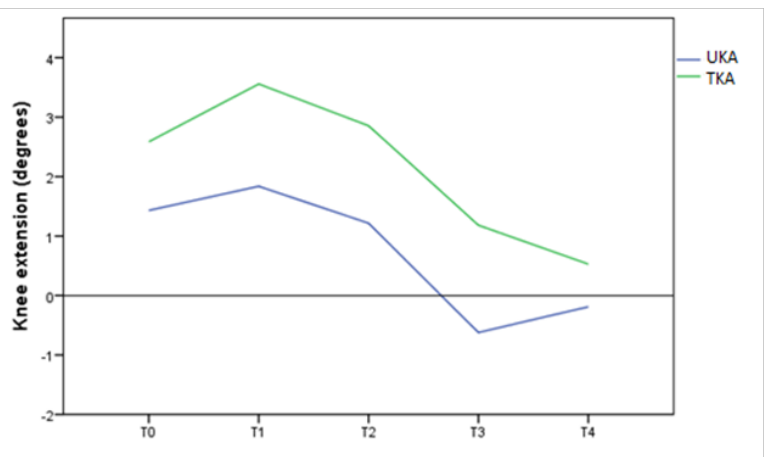

Figure 3 Knee extension expressed in degrees, during multiple measuring moments $(\mathrm{T} 0=$ preoperative, $\mathrm{Tl}=2$ weeks, $\mathrm{T} 2=8$ weeks, $\mathrm{T} 3=6$ months, $\mathrm{T} 4=$ I year)

Table 3 Descriptive statistics of the knee extension preoperative (T0) and one year after the procedure (T4)

\begin{tabular}{lccccc}
\hline & $\mathbf{T}^{0}$ & $\mathbf{T}^{4}$ & $\mathbf{T}^{0}$ & $\mathbf{T}^{4}$ \\
UKA & & & TKA & & \\
\hline N & 60 & 60 & 180 & 180 \\
Minimum & $-5^{\circ}$ & $-10^{\circ}$ & $-10^{\circ}$ & $-5^{\circ}$ \\
Maximum & $15^{\circ}$ & $10^{\circ}$ & $30^{\circ}$ & $10^{\circ}$ \\
Mean & $1^{\circ}$ & $0^{\circ}$ & $3^{\circ}$ & $0^{\circ}$ \\
SD & 4 & 2 & $6^{\circ}$ & $2^{\circ}$ \\
\hline
\end{tabular}

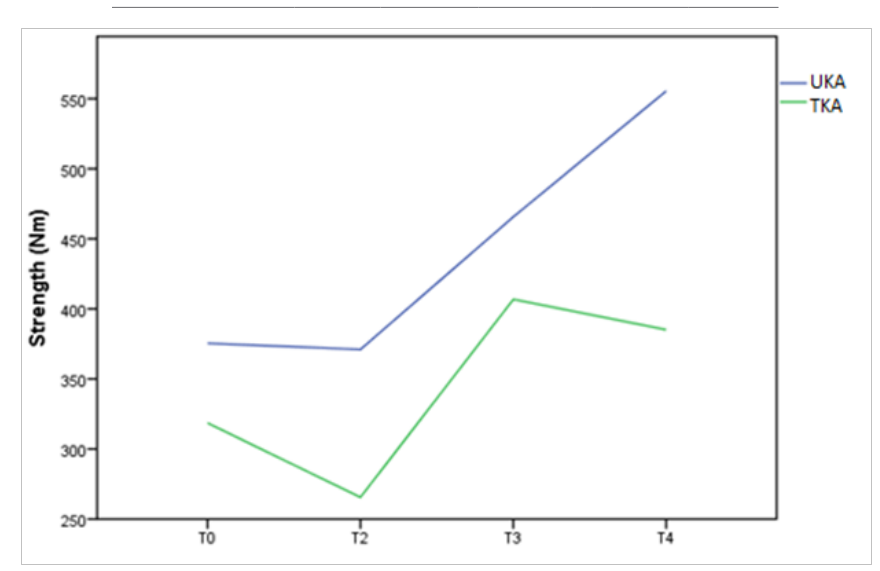

Figure 4 Strength performed during the isometric squad test, during multiple measuring moments $(\mathrm{T} 0=$ preoperative, $\mathrm{Tl}=2$ weeks, $\mathrm{T} 2=8$ weeks, $\mathrm{T} 3=6$ months, $\mathrm{T} 4=\mathrm{I}$ year $)$.

\section{Oxford Knee score}

Preoperative there was no significant difference between the groups during the isometric squad test $(\mathrm{p}=0.984)$. One year postoperative there was no significant difference $(\mathrm{p}=0.913)$ noticeable between the groups (Figure 5, Table 5). Paired sampled t-test showed for both procedures a significant strength improvement (UKA; $\mathrm{p}=0.000$, TKA: $\mathrm{p}=0.000$ ).
Table 4 Descriptive statistics of the isometric strength preoperative (T0) and one year after the procedure (T4)

\begin{tabular}{llllll}
\hline & $\mathbf{T}^{0}$ & $\mathbf{T}^{4}$ & & $\mathbf{T}^{0}$ & $\mathbf{T}^{4}$ \\
UKA & & & TKA & & \\
\hline $\mathrm{N}$ & 60 & 60 & & 180 & 180 \\
Minimum & 29 & $15 \mathrm{I}$ & 30 & 34 \\
Maximum & 900 & 1882 & 1496 & 1075 \\
Mean & 356 & 538 & 322 & 382 \\
SD & 226 & 292 & & 243 & 212
\end{tabular}

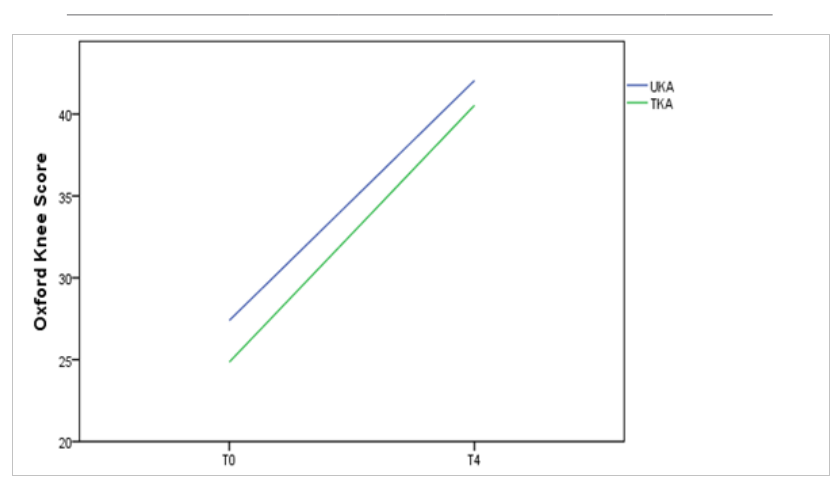

Figure 5 Oxford knee score, during multiple measuring moments ( $\mathrm{T} 0=$ preoperative, $\mathrm{T} 4=$ I year).

Table 5 Descriptive statistics of the Oxford Knee Score preoperative (T0) and one year after the procedure (T4)

\begin{tabular}{lccccc}
\hline & $\mathbf{T}^{\mathbf{0}}$ & $\mathbf{T}^{4}$ & & $\mathbf{T}^{\mathbf{0}}$ & $\mathbf{T}^{4}$ \\
UKA & & & TKA & & \\
\hline $\mathrm{N}$ & 60 & 60 & & 180 & 180 \\
Minimum & 10 & 21 & 5 & 10 \\
Maximum & 44 & 48 & 43 & 48 \\
Mean & 27 & 42 & 25 & 40 \\
SD & 8 & 6 & 8 & 7
\end{tabular}

Complications: Of the total of 240 procedures 7 total knee prostheses needed to be mobilized under anesthetics, due to stiffness of the knee. 2 total knees developed alate onset infection (respectively 2.5 year and 3 years after the implantation) due to haematogenous seeding after a urinary tract infection. In both cases the infection was successfully treated with a combination of open debridement and intravenous antibiotics. 1 total knee exhibited stiffness and patellofemoral malt racking, which needed a revision with an additional patella button.

\section{Discussion}

Postoperative strength loss after TKA has always been a problem, which is most likely associated with preoperative muscle atrophy and reduced voluntary muscle contractions. ${ }^{11}$ Although patients are preoperative and postoperatively stimulated to exercise, patients are unfortunately still unable to achieve comparable strength as healthy individuals, even years after surgery. ${ }^{7,11}$

Not only pain and range of motion are critical for the postoperative outcome of knee Arthroplasty, lower limb strength is equally a 
critical factor in the postoperative outcome. A sufficient extensor mechanism of the lower limb is required for common daily activities such as climbing stairs, riding a bike or rising from a chair. With this knowledge it is obvious that lower limb strength has an affiliation with the functional outcome score. ${ }^{12}$

Strength loss after total knee Arthroplasty is a complex multi factorial problem including pertinent anatomical, technical, and implant-related factors. ${ }^{13}$ It is hypothesized that the reduction in muscle strength after total knee Arthroplasty is probably the result of muscle atrophy caused by disuse before the procedure that has not been recovered after the surgery. ${ }^{12}$ Whereas postoperative physical therapy reported outstanding gain of the strength, it still doesn't match the norms of age-matched healthy individuals. ${ }^{14}$ Now a days younger and more active patients undergo joint arthroplasties, these patients have higher expectations regarding the outcome of the procedure, due to their active lifestyle. Despite the fact that knee arthroplasties are successful procedures regarding the pain and function of the knee, literature supports the fact that patients are not able to resume their level of [sport] activities as they could before, in contrary to patients after a hip Arthroplasty. ${ }^{1,4,15}$

This unfortunate outcome is not seen in all knee arthroplasties. A recent conducted systematic review revealed that patients are more likely to participate in sport activities after UKA rather than after TKA. ${ }^{1}$ A reason for this phenomenon could be that UKA patients have lower limb strength in their operated limb and therefore able to resume their sport activities on their preceding intensity.

This study is the first to compare pre- and post-operatively lower limb strength between the UKA and TKA. The most important finding of this study is that patients receiving an UKA had significant stronger lower limb strength and a better ROM after the procedure, compared to patients receiving TKA.

Due to the improving postoperative functional outcome, the popularity of the Unicondylar knee Arthroplasty is rising. ${ }^{16,17}$ The strict UKA indications by Kozinn and Scott have been widely reported and debated..$^{18}$ The advantages of the UKA compared to TKA includes; smaller implant, less surgical trauma, preservation of both cruciate ligaments and bone stock. These factors could be responsible for a better outcome of the UKA in patient with an active lifestyle..$^{19,20}$ This study backs current literature regarding the range of motion after knee Arthroplasty; patients with a UKA have a better range of motion compared with those after a TKA. . $^{1,6,7,21}$

Literature regarding the Unicondylar knee Arthroplasty and lower limb strength is scare and distinct. Barker et al researched the leg extension power after Unicondylar knee Arthroplasty in 44 patients, after 1 year there was no statistically significant difference in leg extension power between the operated and contra lateral leg. ${ }^{6}$ Fuchs et al. evaluated the isokinetic strength after Unicondylar knee Arthroplasty and healthy control subjects in 17 patients, where $30 \%$ loss of isokinetic strength was observed in patients after an UKA. ${ }^{19}$ Braito et al. compared the knee extensor strength in 32 patients following minimally invasive UKA and minimally-invasive TKA; no significant difference regarding the knee extensor strength was found 8 weeks postoperative. ${ }^{22}$ As these issues are better understood, the UKA may become an even more successful procedure in young and active patients.

\section{Conclusion}

Both UKA and TKA are successful procedures for treating patient with osteoarthritis, but it seems that patients with a UKA have a significant stronger lower limb strength one year after the procedure compared those who received a TKA. ${ }^{23}$ In our quest to restore normal knee function UKA could be a better choice than TKA in patients with anteromedial osteoarthritis. ${ }^{24}$

\section{Acknowledgements}

None.

\section{Conflict of interest}

Author declares there is no conflict of interest in publishing the article.

\section{References}

1. Witjes S, Gouttebarge V, Kuijer PP, et al. Return to Sports and Physical Activity After Total and Unicondylar Knee Arthroplasty: A Systematic Review and Meta-Analysis. Sports Med. 2016;46(2):269-292.

2. Page CJ, Hinman RS, Bennell KL. Physiotherapy management of knee osteoarthritis. Int J Rheum Dis. 2011;14(2):145-151.

3. Van Manen MD, Nace J, Mont MA. Management of primary knee osteoarthritis and indications for total knee arthroplasty for general practitioners. J Am Osteopath Assoc. 2012;112(11):709-715.

4. Ethgen $\mathrm{O}$, Bruyère $\mathrm{O}$, Richy $\mathrm{F}$, et al. Health-related quality of life in total hip and total knee arthroplasty. A qualitative and systematic review of the literature. J Bone Joint Surg. 2004;86-A(5):963-974.

5. Pandit H, Gulati A, Jenkins C, et al. Unicompartmental knee replacement for patients with partial thickness cartilage loss in the affected compartment. Knee. 2011;18(3):168-171.

6. Machner A, Pap G, Awiszus F. Evaluation of quadriceps strength and voluntary activation after unicompartmental arthroplasty for medial osteoarthritis of the knee. J Orthop Res. 2002;20(1):108-111.

7. Barker KL, Jenkins C, Pandit H, et al. Muscle power and function two years after unicompartmental knee replacement. Knee. 2012;19(4):360-364.

8. Altman R, Asch E, Bloch D, et al. Development of criteria for the classification and reporting of osteoarthritis. Classification of osteoarthritis of the knee. Diagnostic and Therapeutic Criteria Committee of the American Rheumatism Association. Arthritis Rheum. 1986;29(8):1039-1049.

9. Xie F, Ye H, Zhang Yu, et al. Extension from inpatients to outpatients: validity and reliability of the Oxford Knee Score in measuring health outcomes in patients with knee osteoarthritis. Int $J$ Rheum Dis. 2011;14(2):206-210.

10. Maffiuletti NA, Bizzini M, Schatt S, et al. A multi-joint lower-limb tracking-trajectory test for the assessment of motor coordination. Neuroscience Letters. 2005;384(1-2):106-111.

11. Stevens JE, Mizner RL, Snyder Mackler L. Quadriceps strength and volitional activation before and after total knee arthroplasty for osteoarthritis. J Orthop Res. 2003;21(5):775-779.

12. Silva M, Shepherd EF, Jackson WO, et al. Knee strength after total knee arthroplasty. J Arthroplasty. 2003;18(5):605-611.

13. Greene KA, Schurman JR. Quadriceps muscle function in primary total knee arthroplasty. J Arthroplasty. 2008;23(7 Suppl):15-19.

14. Walsh M, Woodhouse LJ, Thomas SG, et al. Physical impairments and functional limitations: a comparison of individuals 1 year after total knee arthroplasty with control subjects. Phy Ther. 1998;78(3):248-258.

15. Noble PC, Gordon MJ, Weiss JM, et al. Does total knee replacement restore normal knee function?. Clin Ortho Relat Res. 2005;(431):157-165. 
16. Lygre SH, Espehaug B, Havelin LI, et al. Pain and function in patients after primary unicompartmental and total knee arthroplasty. J Bone Joint Surg Am. 2010;92(18):2890-2897.

17. Iorio R, Healy WL. Unicompartmental arthritis of the knee. J Bone Joint Sur Am. 2003;85-A(7):1351-1364.

18. Kozinn S C, Scott R. Unicondylar knee arthroplasty. J Bone Joint Surg Am. 1989;71(1):145-150.

19. Fuchs S, Frisse D, Laass $\mathrm{H}$, et al. Muscle strength in patients with unicompartmental arthroplasty. Am J Phys Med Rehabil. 2004;83(8):650-4;quiz 655-7.

20. Hanssen AD, Stuart MJ, Scott RD, et al. Surgical options for the middleaged patient with osteoarthritis of the knee joint. Instr Course Lect 2001;50:499-511.
21. Lyons MC, MacDonald SJ, Somerville LE, et al. Clin Orthop Relat Res. 2012;470(1):84-90.

22. Braito M, Giesinger JM, Fischler S, et al. Knee Extensor Strength and Gait Characteristics After Minimally Invasive Unicondylar Knee Arthroplasty vs Minimally Invasive Total Knee Arthroplasty: A Nonrandomized Controlled Trial. J Arthroplastry. 2016;31(8):1711-1716.

23. Essving P, Axelsson K, Kjellberg J, et al. Reduced morphine consumption and pain intensity with local infiltration analgesia (LIA) following total knee arthroplasty. Acta Orthop. 2010;81(3):354-360.

24. Healy WL, Sharma S, Schwartz B, et al. Athletic activity after total joint arthroplasty. J Bone Joint Surg Am. 2008;90(10):2245-2252. 